\title{
Dynamic Fault Tolerant Topology Control for Wireless Sensor Network Based on Node Cascading Failure
}

\author{
https://doi.org/10.3991/ijoe.v14i05.8644 \\ Yang Xiao \\ Hubei University of Education, Wuhan, China \\ bronexy@126. com
}

\begin{abstract}
To address the node cascading failure (CF) of the wireless sensor networks (WSNs), considering such factors as node load and maximum capacity in scale-free topology, this paper establishes the WSN dynamic fault tolerant topology model based on node cascading failure, analyses the relationships between node load, topology and dynamic fault tolerance, and demonstrates the proposed model through simulation test. It studies the effects of topology parameter and load in case of random node failure in the network node cascading failure, and utilizes the theoretical derivation method to derive the structural feature of scale-free topology and the capacity limit for the WSNs large-scale cascading failure, effectively enhancing the cascading fault tolerance of traditional WSNs. The simulation test results show that, with the degree distribution parameter $C$ increasing, the minimum network node degree will increase accordingly, and in highly intensive topology, the dynamic fault tolerance will be better; with the parameter $\lambda$ increasing, the maximum degree of the network node will gradually decrease, and the degree distribution of topology structure tends to be uniform, so that the large-scale cascading failure caused by node failure will have less influence on the WSN, and further improve the dynamic fault tolerance performance of the system.
\end{abstract}

Keywords-Wireless Sensor Networks (WSNs), scale-free topology, dynamic fault-tolerant capacity, cascading failure (CF), optimization

\section{$1 \quad$ Introduction}

The cascading failure (CF) is the key issue that constrains the further development of WSNs. CF means that in case that one node fails in the system, the load of this node would be transferred to the neighbouring nodes, and if the total load of these nodes exceeds the upper limit of its capacity, the neighbouring nodes would also fail, finally causing widespread network $\mathrm{CF}$ in the vicious cycle [1-3].

The fault tolerance performance of WSNs can help restrain the CF of the nodes. The fault tolerance can be mainly divided into two types - static and dynamic fault tolerance. Lots of researches have been made on the static fault tolerance, but the CF of network nodes is related to the dynamic fault tolerance [4-9]. The scale-free topology structure can well restrain the node failure, further enhancing the dynamic fault 
tolerance of WSNs [10-12]. However, the traditional fault tolerance indicates that the failure of a single node will not cause the $\mathrm{CF}$ of neighbouring nodes, which is obviously contrary to the actual situation. So far, such methods as CASCADE model and Sandpile model, etc. have been proposed to restrain the CF of network noded [13-23].

The capacity limit and intrusion tolerance of network nodes is an effective method to restrain CF. According to current studies, optimization has been made mostly to the node load capacity or the link connection between and neighbouring nodes etc. However, the inherent features of WSNs have limited the above optimizations to some extent; therefore, for the network node $\mathrm{CF}$, the analysis should be mainly made on the fault tolerance to scale-free topology cascading failure.

To address the node CF of the wireless sensor networks (WSNs), considering such factors as node load and maximum capacity in scale-free topology, this paper establishes the WSN dynamic fault tolerant topology model based on node cascading failure, analyses the relationships between node load, topology and dynamic fault tolerance, and demonstrates the proposed model through simulation test.

\section{WSN node cascading failure model}

CF in WSNs means that in case that one node fails in the system, the load of this node would be transferred to the neighbouring nodes, and if the total load of these nodes exceeds the upper limit of its capacity, the neighbouring nodes would also fail, finally causing widespread network $C F$ in the vicious cycle. The node load $L_{i}$ is expressed as follows:

$$
L_{i}=k_{i}^{\alpha}
$$

where, $k_{i}$ means the degree of node; $\alpha$ is the load parameter; $L_{i}$ is expressed with the power function of node degree:

$$
L_{i}(\text { new })=L_{i}+\Delta_{i j}=L_{i}+L_{j} / k_{j}
$$

$L_{i}$ (new) is the sum of original load and new load. The CF resulting from load redistribution will not stop until the load of the nodes is less than the capacity. The CF size can be expressed by the residual network connection size:

$$
S=N^{1} / N
$$

$\mathrm{N} 1$ and $\mathrm{N}$ stand for the initial number of network nodes and the maximum number of nodes in the connection size. The higher $\mathrm{S}$ is, the less damage there will be to the network, and the smaller the scale of the CF will be.

Figure 1 shows the topology CF process of a WSN. Based on formula 1-3 and Fig 1, the node CF process of the WSN consists of the following steps:

(1) Set the nodes of a specific WSN as vi $(i=1,2,3 \ldots, N)$, and the load as Li, and keep all nodes in the initial state with the same capacity $\mathrm{C}$; 
(2) Suppose that after some time of operation, one node vi of the network system fails, and distributes its load in a balanced manner to a neighbouring node $\mathrm{K}$, then the load of the neighbouring node $\mathrm{vj}$ is:

$$
L_{i}(\Delta)=L_{i}+L_{j} / k_{j}
$$

kj means the degree of node vj; and $\operatorname{Li}(\Delta)$ means the re-distributed load.

(3) Evaluate the distributed loads of all residual nodes. If $\operatorname{Li}(\Delta)>C$, this node fails; continue to perform calculation in step 2 until $\operatorname{Li}(\Delta)<\mathrm{C}$ for all nodes, and then the $\mathrm{CF}$ process is finished.

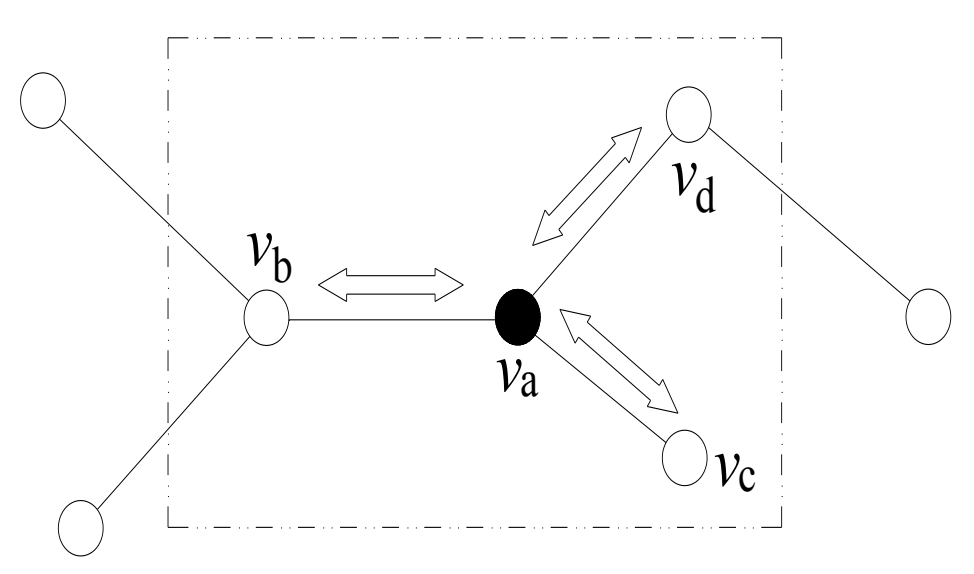

Fig. 1. Schematic diagramof WSN cascading failure

\section{WSN CF fault tolerance evaluation}

Considering that the WSN node capacity is pre-set in manufacturing process, it is not realistic to use the dynamic optimization method to restrain the CF of scale-free topology. Suppose the initial capacity of nodes is $C$ and that the load is $\alpha$ : when $a$ is small, the node load should also be small; therefore, there is no CF in the WSN, and the maximum connection size $S$ in the network system is large; with $\alpha$ increasing gradually, the node load changes greatly, and accordingly CF occurs more easily; when the maximum connection component $S$ decreases to the minimum standard $S_{t h}$, $\alpha$ will be the limit value for the WSN cascading failure.

The probability generating function is used to calculate the value $S$ in cascading failure, and the degree distribution function of the WSN scale-free topology is expressed as:

$$
p(k)=c k^{-\lambda}(c>0, \lambda>0)
$$

Then the probability generating function is expressed as: 


$$
g_{0}(x)=\sum_{k=k_{\min }}^{k_{\max }} p(k) x^{k}
$$

$k_{\min }$ and $k_{\max }$ mean the upper and lower limits for node degree. Let $\mathrm{q}^{1}(\mathrm{k})$ be the probability of one failure node, and select any edge connected to the failure node to make load transmission. The generating function of the node transmitting the load can be expressed as:

$$
g_{1}(x)=\sum_{k=k_{\min }-1}^{k_{\max }-1} q^{1}(k) x^{k}=\frac{1}{x} \sum_{k=k_{\min }}^{k_{\max }} q^{1}(k) x^{k}
$$

In actual network system, the initial loads of all nodes differ, and the failure nodes have different influences on the neighbouring nodes; therefore, $\mathrm{q}^{1}(\mathrm{k})$ can be expressed as:

$$
q^{1}(k)=\sum_{i=k_{\min }}^{k_{\max }} e_{i k}=\sum_{i=k_{\min }}^{k_{\max }} p_{1}(i) p_{2}(k) p_{3}(i k)
$$

$e_{i k}$ indicates the probability that the load of the failure node is transmitted to the second neighbouring nodes and then causes its failure; $P_{2}(k)$ is the probability that one edge is randomly selected to the failure node.

$$
p_{2}(k)=k p(k) / \sum_{k=k_{\min }}^{k_{\max }} k p(k)
$$

$\mathrm{p}_{3}(\mathrm{ik})$ means the probability of causing the node failure of $K$ node degree.

$$
p_{3}(i k)=\frac{i^{\alpha} / i}{c_{0}-k^{\alpha}}, \alpha<\frac{\ln \frac{c_{0}}{1+k_{\max }^{-1}}}{\ln k_{\max }}
$$

Then $\mathrm{q}^{1}(\mathrm{k})$ can be transformed into:

$$
q^{1}(k)=\sum_{i=k_{\min }}^{k_{\max }} c i^{-\lambda} \frac{k c k^{-\lambda}}{\sum_{i=k_{\min }}^{k_{\max }} k c k^{-\lambda}} \frac{i^{\alpha} / i}{c_{0}-k^{\alpha}}=\sum_{i=k_{\min }}^{k_{\max }} \frac{c(2-\lambda) i^{\alpha-\lambda-1} k^{1-\lambda}}{\left(k_{\max }^{2-\lambda}-k_{\min }^{2-\lambda}\right)\left(c_{0}-k^{\alpha}\right)}, \alpha<\frac{\ln \frac{c_{0}}{1+k_{\max }^{-1}}}{\ln k_{\max }}
$$

Given that the remaining degree (arriving at the node) is (RD) arrive, and that the network cascading failure is $(\mathrm{CF})_{\mathrm{i}}$, when one node fails in the system, the connected 
node on the edge $K$ will also fail, and then the maximum number of connected normal nodes in the network system may fall into different cases depending on the remaining degree: (1) when $(\mathrm{RD})_{\text {arrive }}=0,(\mathrm{CF})_{1}=1$; (2) when $(\mathrm{RD})_{\text {arrive }}=1,(\mathrm{CF})_{2}=1+(\mathrm{CF})_{1}$, and so on. The generating function $\mathrm{h}_{1}(\mathrm{x})$ of the network size after $\mathrm{CF}$ is:

$$
h_{1}(x)=x q^{1}(0)+x q^{1}(1) h_{1}(x)+x q^{1}(2)\left[h_{1}(x)\right]^{2}+\mathrm{L}=x g_{1}\left(h_{1}(x)\right)
$$

Combining $\mathrm{g}_{1}(\mathrm{x})$ with $\mathrm{q}^{1}(\mathrm{k})$, we have:

$$
g_{1}\left(h_{1}(x)\right)=\sum_{k=k_{\min }}^{k_{\max }} q^{1}(k)\left[h_{1}(x)\right]^{k-1}=\sum_{k=k_{\min }}^{k_{\max }} \sum_{i=k_{\min }}^{k_{\max }} \frac{c(2-\lambda) i^{\alpha-\lambda-1} k^{1-\lambda}}{\left(k_{\max }^{2-\lambda}-k_{\min }^{2-\lambda}\right)\left(c_{0}-k^{\alpha}\right)}\left[h_{1}(x)\right]^{k-1}, \alpha<\frac{\ln \frac{c_{0}}{1+k_{\max }^{-1}}}{\ln k_{\max }}
$$

When $\mathrm{x}=1, \mathrm{~h}_{1}(1)$ can be transformed into:

$$
h_{1}(1)=g_{1}\left(h_{1}(1)\right)=\sum_{k=k_{\min }}^{k_{\max }} \sum_{i=k_{\min }}^{k_{\max }} \frac{c(2-\lambda) i^{\alpha-\lambda-1} k^{1-\lambda}}{\left(k_{\max }^{2-\lambda}-k_{\min }^{2-\lambda}\right)\left(c_{0}-k^{\alpha}\right)} \times\left[h_{1}(1)\right]^{k-1}, \alpha<\frac{\ln \frac{c_{0}}{1+k_{\max }^{-1}}}{\ln k_{\max }}
$$

Then the network size in case of node failure is:

$$
h_{0}(1)=\sum_{k=k_{\min }}^{k_{\max }} p(k)[u]^{k}
$$

Based on the network size, the maximum connected component $S$ can be further obtained:

$$
\begin{aligned}
& S=1-h_{0}(1)=1-\sum_{k=k_{\min }}^{k_{\max }} p(k)[u]^{k} \\
& u=\sum_{k=k_{\min }}^{k_{\max }} \sum_{\min }^{k_{\max }} \frac{c(2-\lambda) i^{\alpha-\lambda-1} k^{1-\lambda}}{\left(k_{\max }^{2-\lambda}-k_{\min }^{2-\lambda}\right)\left(c_{0}-k^{\alpha}\right)}[u]^{k-1}, \alpha<\frac{\ln \frac{c_{0}}{1+k_{\max }^{-1}}}{\ln k_{\max }}
\end{aligned}
$$

The two formulas above are the quantified forms of $S$ for CF. Given the electricitysaving capacity and WSN distribution index, $S$ is only related to $\alpha$, that is, the higher $\alpha$ is, the greater $u$ will be; and the maximum connected component $S$ is inversely proportional to load.

The network data transmission quality and service quality are related to $S$; therefore, the data acquisition and quality transmission can be controlled by adjusting the $S$ 
value. The limit capacity of WSN scale-free topology should meet the following requirements:

$$
S_{\mathrm{th}}=1-\sum_{k=k_{\min }}^{k_{\max }} p(k)[\tilde{u}]^{k}, \tilde{u}=\sum_{k=k_{\min }}^{k_{\max }} \sum_{\min }^{k_{\max }} \frac{c(2-\lambda) i^{\tilde{\alpha}-\lambda-1} k^{1-\lambda}}{\left(k_{\max }^{2-\lambda}-k_{\min }^{2-\lambda}\right)\left(c_{0}-k^{\tilde{\alpha}}\right)}[\tilde{u}]^{k-1}, \tilde{\alpha}<\frac{\ln \frac{c_{0}}{1+k_{\max }^{-1}}}{\ln k_{\max }}
$$

As $0<\mathrm{S}_{\mathrm{th}}<1$ and $u$ is under the monotone increasing trend, when $\alpha$ goes towards negative infinity, $u$ tends to be 0 . Hence, in WSN, the widespread CF issue can be restrained by keeping the node load lower than the limit capacity, thus further improving the dynamic fault tolerance of the network system.

\section{Simulation test and analysis}

In the simulation test, the capacity limit of nodes was imposed to boost node failure, and a random node failure system of WSN scale-free topology structure was established. By analysing the relationship between the degree distribution and CF in WSN, the author studied the inhibiting effect of the established model on the node CF.

With Matlab as the simulation platform, two topology structures were constructed to help analyse the validity of the established model. The number of nodes in topology structure 1 was 200 , with the degree distribution $\mathrm{p}(\mathrm{k})=1.89 \mathrm{k}^{-2.89}$, and the number of nodes in topology structure 2 was 200 with degree distribution $\mathrm{p}(\mathrm{k})=2.13 \mathrm{k}^{-2.61}$. Nodes were randomly deleted in the calculation. For the simulation results, please refer to Figure 2, where the $\mathrm{X}$-axis represents the load parameter $\gamma$, and the $\mathrm{Y}$-axis stands for the maximum connected size. The figure shows that as $\gamma$ increased gradually, the CF sizes of both topology structures were on an increasing trend; and the cascading failure of topology structure 2 is more serious than 1 . In topology structure 1 , no widespread node failure occurs, but in 2 , when $\gamma=0.9-1.0$, there occurs widespread node $\mathrm{CF}$, which means that the actual degree distribution of WSN scale-free topology differs greatly from the theoretical calculation result; the theoretical calculation result reflects the statistical average, while the simulation result is only one of the theoretical results.

In the widespread node failure for WSN system, if there still exists a large $S$, then it indicates better WSN scale-free topology fault tolerance. Figure 3 indicates the CF sizes at different values of the degree distribution parameter $C$. The number of nodes was set at 200, and $\mathrm{C}$ was set at 2, 8, and 18 respectively, with the full line in figure indicating the theoretical degree distribution curves at different $C$ values and the scattered points indicating the practical degree distributions at different $C$ values. 


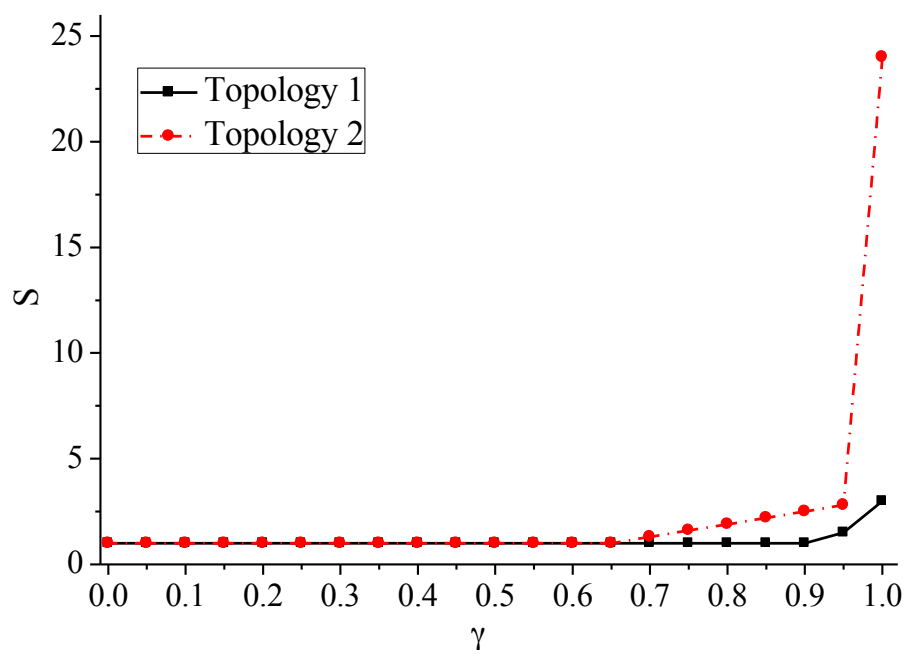

Fig. 2. Simulation test with two kinds of topology structures

Besides, the algorithm was based on the degree distribution adjusted (DDA) controlled by scale-free fault tolerant topology. The figure shows that, with $C$ increasing, the minimum degree $D_{\text {min }}$ gradually increased; when $\mathrm{c}=2, D_{\min }=1$; at $\mathrm{c}=8, D_{\min }=2$; and at $\mathrm{c}=18, D_{\min }=3$, which means that the value $C$ and the node-connected edge has a proportional relation, i.e. the higher $C$ is, the more intensive the topology will be.

Figure 4 depicts the relation curve of the maximum connection size $S$ and the load $\alpha$ at three different values of $C$. With 0.1 as the step size, $\alpha$ gradually increased, and the initial node capacity was set at 15 . It shows that, given $C, S$ was constant no matter how $\alpha$ changed; with $C$ increasing, $S$ was larger,- specifically, when c=2, $S=0.79$; when c=8, $S=0.97$; and when $\mathrm{c}=18, S=0.98$. According to Fig 3 and $4, C$ is related to the intensity of the network topology, i.e. the higher $C$ is, the more intensive the topology structure will be, and the better the dynamic fault tolerance will be.

Figure 5 indicates the CF sizes at different values of another degree distribution parameter $\lambda$. The number of nodes was also set at 200 , and $\lambda$ was set at $2.5,3$, and 3.5 respectively, with the full line in Figure indicating the theoretical degree distribution curves at different $\lambda$ values and the scattered points indicating the practical degree distributions at different $\lambda$ values. Besides, the $\mathrm{X}$-axis represents the node degree $\mathrm{k}$, and the $y$-axis, the node degree distribution function value. The figure shows that with $\lambda$ increasing, the maximum degree $D_{\max }$ gradually increased; when $\lambda=2.5, D_{\max }=16$; when $\lambda=3, D_{\max }=13$; and when $\lambda=3.5, D_{\min }=12$, which means that the higher $\lambda$ is, the fewer nodes there will be with greater node degrees in the network system, and the more uniform the degree distribution of the topology structure tends to be. 


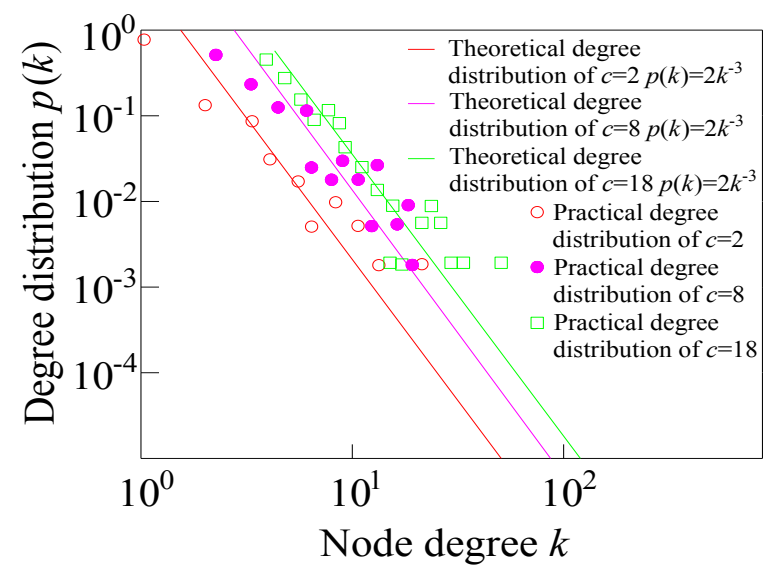

Fig. 3. Degree distribution curves of scale-free topology structures at different distribution parameters

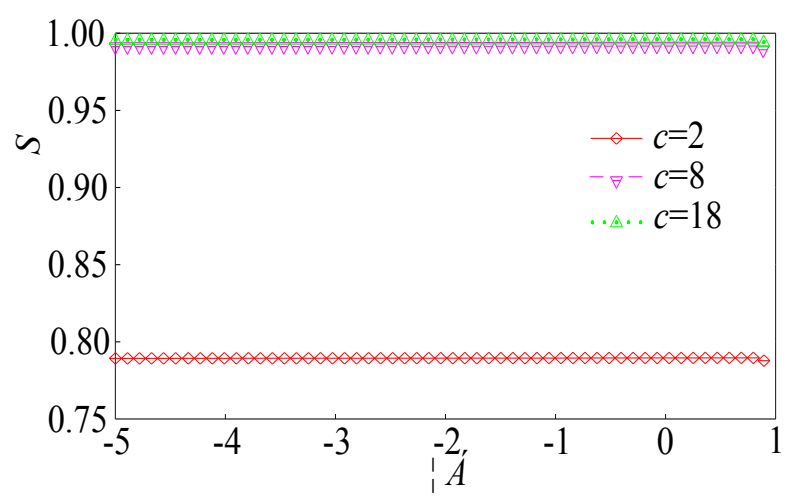

Fig. 4. S- $\alpha$ curve with different distribution parameter

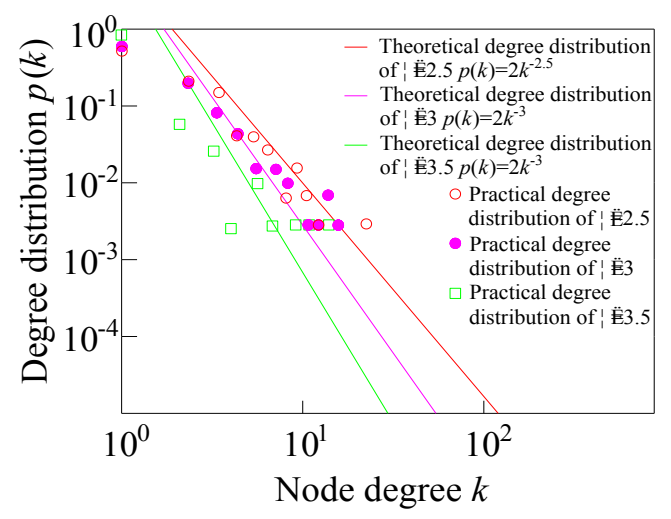

Fig. 5. Degree distribution curves of scale-free topology structures at different $\lambda$ values 
Figure 6 depicts the relation curve of the maximum connection size $S$ and the load $\alpha$ at three different values of $\lambda$. With 0.1 as the step size, $\alpha$ gradually increased, and the initial node capacity was set at 15 . It is found that at different $\lambda$ values, the $S-\alpha$ distribution curve showed the same changing pattern as $C$, i.e. given $\lambda, S$ was constant no matter how $\alpha$ changed; with $C$ increasing, $S$ was larger - specifically, when $\lambda=2.5$, $S=0.70$; when $\lambda=3, S=0.79$; when $\lambda=3.5, S=0.84$. The higher $\lambda$ is, the more uniform the degree distribution of the topology structure will be; otherwise, the more disorderly it will be. In the uniform topology structure distribution, the large-scale cascading failure caused by node failure will have little influence on the WSN, which will further improve the dynamic fault tolerance of the system.

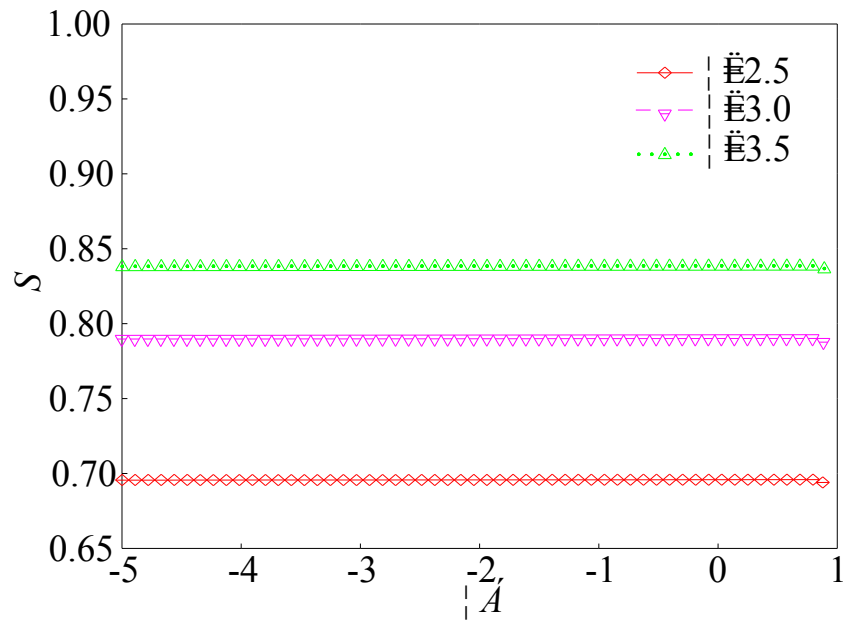

Fig. 6. $S$ - $\alpha$ curves at different $\lambda$ values

\section{Conclusion}

To address the node CF of WSNs, considering such factors as node load and maximum capacity in scale-free topology, this paper establishes the WSN dynamic fault tolerant topology model based on node cascading failure, analyses the relationships between node load, topology and dynamic fault tolerance, and demonstrates the proposed model through simulation test. The following conclusions are obtained:

It studies the effects of topology parameter and load in case of random node failure in the network node cascading failure, and utilizes the theoretical derivation method to derive the structural feature of scale-free topology and the capacity limit for the WSNs large-scale cascading failure, effectively enhancing the cascading fault tolerance of traditional WSNs.

The simulation test results show that, with the degree distribution parameter $C$ increasing, the minimum network node degree will increase accordingly, and in highly intensive topology, the dynamic fault tolerance will be better; with the parameter $\lambda$ increasing, the maximum degree of the network node will gradually decrease, and the 
degree distribution of topology structure tends to be uniform, so that the large-scale cascading failure caused by node failure will have less influence on the WSN, and further improve the dynamic fault tolerance performance of the system.

\section{Acknowledgment}

This work was supported by the Hubei Provincial Department of Education, China under Grant B2017209.

\section{$7 \quad$ References}

[1] Motter, A.E., Lai, Y.C. (2002). Cascade-based attacks on complex networks. Physical Review E Statistical Nonlinear \& Soft Matter Physics, 66(2), 065102. https://doi.org/10.1103/PhysRevE.66.065102

[2] Li, H.M., Liu, H.H. (2017). The optimizated study on preparation process of nano tire pressure sensor used in auto, Academic Journal of Manufacturing Engineering, 15(1), 97104.

[3] Dou, B.L., Wang, X.G., Zhang, S.Y. (2010). Robustness of networks against cascading failures. Physica A Statistical Mechanics \& Its Applications, 389(11), 2310-2317. https://doi.org/10.1103/PhysRevE.77.056103

[4] Boccaletti, S., Latora, V., Moreno, Y., Chavezf, M., Wang D.U.H. (2007). Complex networks: structure and dynamics. Complex Systems \& Complexity Science, 424(4-5), 175308.

[5] Crucitti, P., Latora, V., Marchiori, M. (2003). Model for cascading failures in complex networks. Physical Review E Statistical Nonlinear \& Soft Matter Physics, 69(4 Pt 2), 045104. https://doi.org/10.1103/PhysRevE.69.045104

[6] Crucitti, P., Latora, V., Marchiori, M., Rapisarda, A. (2002). Efficiency of scale-free networks: error and attack tolerance. Physica A Statistical Mechanics \& Its Applications, 320(C), 622-642. https://doi.org/10.1016/S0378-4371(02)01545-5

[7] Motter, A.E. (2004). Cascade control and defense in complex networks. Physical Review Letters, 93(9), 098701. https://doi.org/10.1103/PhysRevLett.93.098701

[8] Zhu, H., Luo, H., Peng, H., Li, L., Luo, Q. (2009). Complex networks-based energyefficient evolution model for wireless sensor networks.Chaos Solitons \& Fractals, 41(4), 1828-1835. https://doi.org/10.1016/j.chaos.2008.07.032

[9] Chen, L.S., Guo, Z.S. (2015). Research on ascs system fault diagnosis of automobile automated mechanical transmission, Academic Journal of Manufacturing Engineering, 14(4), 25-33.

[10] Heide, D., Schäfer, M., Greiner, M. (2008). Robustness of networks against fluctuationinduced cascading failures. Physical Review E Statistical Nonlinear \& Soft Matter Physics, 77(5 Pt 2), 056103. https://doi.org/10.1103/PhysRevE.77.056103

[11] Wang, J., Rong, L., Zhang, L., Zhang, Z. (2008). Attack vulnerability of scale-free networks due to cascading failures. Physica A Statistical Mechanics \& Its Applications, 387(26), 6671-6678. https://doi.org/10.1016/j.physa.2008.08.037

[12] Dai, F., Wu, J. (2006). On constructing, k -connected, k -dominating set in wireless ad hoc and sensor networks, Journal of Parallel \& Distributed Computing, 66(7), 947-958. https://doi.org/10.1016/j.jpdc.2005.12.010 
Paper-Dynamic Fault Tolerant Topology Control for Wireless Sensor Network Based on Node Cascad...

[13] Albert, R., Barabasi, A.L. (2000). Dynamics of complex systems: scaling laws for the period of boolean networks. Physical Review Letters,84(24), 5660. https://doi.org/10.1103/ PhysRevLett.84.5660

[14] Dobson, I., Carreras, B.A., Newman, D.E. (2005). A loading-dependent model of probabilistic cascading failure. Probability in the Engineering \& Informational Sciences, 19(1), 1532. https://doi.org/10.1017/S0269964805050023

[15] Carreras, B.A., Lynch, V.E., Dobson, I., Newman, D.E. (2004). Complex dynamics of blackouts in power transmission systems. Chaos, 14(3), 643-652. https://doi.org/10.1063/ 1.1781391

[16] Goh, K.I., Lee, D.S., Kahng, B., Kim, D. (2005). Cascading toppling dynamics on scalefree networks. Physica A Statistical Mechanics \& Its Applications, 346(1), 93-103. https://doi.org/10.1016/j.physa.2004.08.054

[17] Lee, D.S., Goh, K.I., Kahng, B., Kim, D. (2004). Sandpile avalanche dynamics on scalefree networks. Physica A Statistical Mechanics \& Its Applications, 338(1), 84-91. https://doi.org/10.1016/j.physa.2004.02.028

[18] Lee, S., Younis, M. (2010). Recovery from multiple simultaneous failures in wireless sensor networks using minimum steiner tree. Journal of Parallel \& Distributed Computing, 70(5), 525-536. https://doi.org/10.1016/j.jpdc.2009.12.004

[19] Gonçalves, C. P. (2017). Quantum neural machine learning: backpropagation and dynamics, NeuroQuantology, 15(1), 22-41. https://doi.org/10.14704/nq.2017.15.1.1008

[20] Li, Z.J. (2017). Application of neural network technology in machining error recovery, Journal of Manufacturing Engineering, 15(3), 6-11.

[21] Banerjee, S., Ghosh, A., Mitra S.K. (2017). A modified mathematical model for lifetime enhancement in wireless sensor network, Mathematical Modelling of Engineering Problems, 4(2), 84-90. https://doi.org/10.18280/mmep.040204

[22] Fichera, A., Frasca, M., Volpe, R. (2017). The centralized energy supply in a network of distributed energy systems: A cost-based mathematical approach, International Journal of Heat and Technology, 35(S1), S191-S195. https://doi.org/10.18280/ijht.35Sp0127

[23] Fichera, A., Frasca, M., Volpe, R. (2016). On energy distribution in cities: a model based on complex networks, International Journal of Heat and Technology, 34(4), 611-615. https://doi.org/10.18280/ijht.340409

\section{Author}

Yang Xiao (bronexy@126.com) received the M.S. degree in communication engineering from Wuhan University of Technology, Wuhan, China, in 2004. He is currently a $\mathrm{PhD}$ candidate in the School of Automotive Engineering at Wuhan University of Technology, and also a Senior Lecturer of the School of Physics and Mechanical \& Electrical Engineering at Hubei University of Education, Wuhan. His research interests include MAC protocols and routing algorithms for WSNs and VANETs.

Article submitted 30 March 2018. Final acceptance 05 May 2018. Final version published as submitted by the author. 